\title{
Carpal tunnel syndrome
}

\author{
Jeremy D P Bland ${ }^{1}$
}

Kent and Canterbury Hospital,

Canterbury CT1 3NG

Jeremy.Bland@ekht.nhs.uk

BMJ 2007:335:343-6

doi:10.1136/bmj.39282.623553.AD

\section{Why should I read this article?}

Carpal tunnel syndrome (CTS) is the commonest peripheral nerve problem in the United Kingdom and has considerable employment and healthcare costs. If recognised early it is readily treatable. No established UK guidelines exist for diagnosis and management, but the American Academy of Neurology issued guidelines in 1993, which remain current as no major recent advances have occurred. ${ }^{1}$

\section{What causes carpal tunnel syndrome?}

Carpal tunnel syndrome results from compromise of median nerve function at the wrist caused by increased pressure in the carpal tunnel, an anatomical compartment bounded by the bones of the carpus and the transverse carpal ligament. Although the ends of the tunnel are in free communication with the surrounding tissues, tissue pressure in the tunnel is much higher in patients with CTS (32-110 $\mathrm{mm} \mathrm{Hg}$, depending on wrist position) than in patients with normal wrists $(2-31 \mathrm{~mm}$ $\mathrm{Hg}^{2}$ Pressures are raised by wrist flexion and extension, and finger flexion.

Intermittent or sustained high tissue pressure in the tunnel impairs microvascular circulation in the median nerve and leads to spurious generation of action potentials, local demyelination, and ultimately axonal loss. It may also stimulate the proliferation of subsynovial connective tissue in the tunnel, according to pathological studies of CTS. ${ }^{3}$ Anything that reduces the dimensions of the tunnel or increases the volume of its contents will predispose to CTS, and many medical associations have been reported, but most cases are idiopathic.

A study of 4488 individuals recruited from the St Thomas' UK adult twin registry found genetic predisposition to be the single strongest factor in predicting the development of the syndrome. ${ }^{4}$ Obesity is a risk factor in younger patients. ${ }^{5}$ The role of occupational and recreational hand use in causation remains controversial. If overuse of the hands does contribute, it may be a relatively minor factor, though most patients report that heavy use of the hands aggravates the symptoms. A Scandinavian survey found population prevalences of $14.4 \%$ for median nerve distribution paraesthesias, $3.8 \%$ for CTS diagnosed on clinical grounds, $4.9 \%$ for neurophysiological focal impairment of the median nerve at the wrist, and $2.7 \%$ for neurophysiologically confirmed clinical CTS. ${ }^{6}$
Incidence peaks in the late 50 s, particularly in women, and the late 70s, when the sex ratio is more equal. ${ }^{7}$ It is also common, transiently, in late pregnancy. Elderly people tend to present with more severe CTS for the same length of history, with 59\% of patients aged over 65 having thenar atrophy at presentation compared with $18 \%$ of younger patients. ${ }^{8}$ CTS in older patients is easily confused with other, less treatable, disorders.

\section{When should I suspect CTS?}

Although the syndrome encompasses a range of severity (from transient subjective sensory symptoms to irreversible thenar wasting and sensory loss) it should be recognised before permanent deficits develop. Patients woken by paraesthesias or pain - the distribution of which includes median nerve territory (the thumb and first two and a half fingers) - have CTS until proved otherwise. Some patients will also complain of sensory disturbance in the whole hand or pain radiating up the arm to the shoulder. Patients whose paraesthesias are limited to the ulnar side of the hand are unlikely to have CTS. ${ }^{9}$ About $55 \%-65 \%$ of cases are bilateral at first presentation and most patients present first with the dominant hand. ${ }^{7}$

Daytime symptoms may be noticed with particular activities, particularly those that involve holding the arms raised. Patients may complain of a perception of swelling of the hand or fingers, but visible swelling is rare and should prompt consideration of other conditions with secondary CTS. Sensory loss in median nerve territory and weakness and wasting of the median innervated thenar muscles are reliable but late indicators of CTS.

The American Academy of Neurology's guidelines state that the likelihood of a diagnosis increases with the number of standard symptoms and provocative factors present (box 1). The most widely used provocative physical tests are Phalen's sign (the provocation of median paraesthesias by flexion of the wrist to $90^{\circ}$ for 60 seconds) and Tinel's sign (the provocation of paraesthesias by tapping over the carpal tunnel). These

\section{SOURCES ANDS SELECTION CRITERIA}

Evidence for the efficacy of treatment comes mainly from reviews conducted by the Cochrane

Collaboration. ${ }^{16-18} \mathrm{I}$ also searched Medline, used a personal reference archive, and analysed the computerised records of over 20000 patients with suspected carpal tunnel syndrome in east Kent 


\section{Box 1| Standard symptoms of carpal tunnel syndrome*}

- Dull, aching discomfort in the hand, forearm, or upper arm

- Paraesthesias in the hand

-Weakness or clumsiness of the hand

- Dry skin, swelling, or colour changes in the hand

- Occurrence of any of the above in the median distribution

- Provocation of symptoms by sleep

- Provocation of symptoms by sustained hand or arm positions

- Provocation of symptoms by repetitive actions of the hand or wrist

- Mitigation of symptoms by changing hand posture or shaking the wrist

${ }^{*}$ According to the American Academy of Neurology's guidelines

signs have been compared with nerve conduction studies as a diagnostic gold standard in many studies. In such studies, Phalen's sign has sensitivity ranging from $10 \%$ to $73 \%$ and specificity from $55 \%$ to $86 \%$. Tinel's sign has sensitivity ranging from $8 \%$ to $100 \%$ and specificity from $55 \%$ to $87 \%$, the wide ranges probably reflecting the difficulty in standardising the test methods. Both signs are less reliable in advanced CTS. ${ }^{10}$

On general examination of the patient, be alert for signs of endocrine disease and connective tissue disorders, which can predispose to CTS, and other hand problems such as Raynaud's phenomenon, vibration white finger, trigger finger, and Dupuytren's contracture, which can all coexist with CTS. Many conditions have been mistaken for CTS; box 2 lists some important differential diagnoses.

\section{What laboratory investigations are needed?}

Plain $\mathrm{x}$ ray examination of the hand is not cost effective in idiopathic CTS - an American study concluded that costs were $\$ 5869$ (£2850; €4250) to $\$ 20115$ for each finding of therapeutic significance. ${ }^{11}$ A check for diabetes, however, is inexpensive and appropriate. Most patients with CTS will be in an age group where

\section{Box 2 Conditions that may be confused with carpal tunnel syndrome}

- Cervical radiculopathy (especially C6/7)-look for local neck pain on movement and neurological signs outside the territory of the distal median nerve

- Ulnar neuropathy-this can also produce nocturnal paraesthesias; the distribution will usually be to the medial side of the hand.

- Raynaud's phenomenon-this should be recognisable from a history of symptoms related to cold exposure

- Vibration white finger-suspect this if the patient uses vibrating hand tools at work

- Osteoarthritis of the metacarpophalangeal joint of the thumb-this can produce a spurious appearance of thenar wasting but not true weakness or sensory deficit

- Tendonitis-specific tests may help in diagnosis, such as Finkelstein's test for De Quervain's tenosynovitis

- Generalised peripheral neuropathies-these should be recognised from the wider distribution of symptoms and reflex changes

- Motor neurone disease-this can present with wasting in one hand but does not produce sensory symptoms

- Syringomyelia-features such as prominent loss of temperature sensation in the hands should give a clue

- Multiple sclerosis-this should be recognised from the presence of neurological abnormalities disseminated in location and time undetected hyperglycaemia is common. The value of blood tests to screen for connective tissue disease and thyroid function is uncertain, particularly in the absence of any clinical indication other than CTS. ${ }^{12}$

Complex investigations are not necessary before starting conservative treatment in clinically obvious cases. However, in cases of diagnostic doubt, and before surgery, nerve conduction studies should be carried out. The American Academy of Neurology's guidelines suggest electrodiagnostic studies and therapeutic trials with non-invasive treatment as the strategies of choice when clinical diagnosis is uncertain. Nerve conduction studies should include sufficient measurements to localise median nerve dysfunction to the carpal tunnel, evaluate its severity, and exclude more widespread neuropathy. ${ }^{13}$ Neurophysiological severity of CTS can be expressed on the 7 point Canterbury scale $(0=$ no abnormality, $6=$ no recordable median motor or sensory potentials), which I have shown correlates with surgical prognosis. ${ }^{14}$

However, nerve conduction studies have a small false negative rate; a precise figure is not available for this because no better test exists for comparison as a gold standard. However, with modern nerve conduction studies, it is probably around 5-10\%. In east Kent, $4.3 \%$ of 3544 successful carpal tunnel decompressions had normal preoperative nerve conduction studies (unpublished personal data).

Conversely not all patients with a neurophysiologically demonstrated median neuropathy at the wrist necessarily have symptoms related to that. An alternative, or ideally complementary, approach is provided by high resolution ultrasonography of the median nerve. In a blind comparison with nerve conduction studies as a gold standard, ultrasonography achieved $89 \%$ sensitivity and $69 \%$ specificity. ${ }^{15}$ However, this study retrospectively optimised the ultrasound measurement cut-off values to achieve the best possible diagnostic performance and is limited in any case by the lack of an absolute diagnostic standard for comparison.

Magnetic resonance imaging can make similar measurements of median nerve dimensions but is more expensive. Ultrasonography is more comfortable than nerve conduction studies for patients but will not detect other nerve problems that may be contributing to the presentation. Ultrasonography may show unsuspected structural abnormalities of relevance, such as bifid median nerves, persistent median arteries, or space occupying lesions in the tunnel, but these are rare and even more rarely do they dictate alternative management.

\section{How is CTS treated?}

Many treatments, both conventional and complementary, have been suggested for CTS (box 3). Few are supported by good quality evidence from randomised controlled trials. The available studies have been well summarised in a series of reviews by the Cochrane Collaboration. ${ }^{16-19}$ 
The recommendations of the American Academy of Neurology for treatment remain reasonable. The academy suggests splinting, activity modification, and non-steroidal anti-inflammatory drugs - and possibly diuretics if there is limb swelling-as conservative treatment, followed by steroid injection and surgery if these fail or in patients with progressive motor deficit. However, activity modification, diuretics, and nonsteroidal anti-inflammatory drugs have no positive support from any randomised trials. Meta-analysis of several randomised trials shows that vitamin B-6 supplementation has a negligible therapeutic effect. ${ }^{19}$

The purpose of treatment is to alleviate the symptoms and, in some people, prevent worsening of the condition. A few, mostly elderly, patients have thenar wasting but no symptoms. Little is to be gained from surgery in such cases. CTS is not necessarily progressive. The condition in some patients may fluctuate slightly for many yearswith more symptoms during periods of heavy hand use or variation with the seasons-without progressing to irreversible median nerve damage. The condition may even remit spontaneously. In one study $23 \%$ of participants improved over 12-15 months without active intervention though this was not a randomised trial and the researchers were not able to rigorously control factors such as activity modification instituted by the patients. ${ }^{20}$ The potential benefits of treatment must be viewed against this background. Only three treatments are supported by a substantial body of experimental evidence: splinting, steroids, and surgery.

\section{Splinting}

A removable wrist brace that maintains the wrist at a neutral angle without applying direct compression over the carpal tunnel provides mechanical respite for the nerve. Such supports are often too cumbersome for daytime use, but for those patients who tolerate them at night, they are often an effective way of achieving an undisturbed night's sleep. In a trial comparing splinting with surgery, $37 \%$ of patients in the splint group obtained

\section{Box 3 Suggested treatments}

\section{Treatments supported by high quality evidence}

- Splinting ${ }^{\star 16}$

- Steroids ${ }^{17}$ —oral, local injection, local iontophoresis

- Surgical decompression ${ }^{1618}$ —open (several variations, with or without tenosynovectomy, transverse carpal ligament reconstruction, and external/internal neurolysis; all seem equally effective with no clear evidence to support the use of the more elaborate procedures); endoscopic (one or two portal)

\section{Other treatments}

- Diuretics*

- Yoga

- Non-steroidal anti-inflammatory drugs*

- Ultrasonography

- Rest or activity modification*

- Acupuncture

- Nerve and tendon gliding exercises

- Serratiopeptidase

- Vitamin B-6

- Magnet therapy

- Synovectomy only

- Cognitive behaviour therapy

- Chiropractic manipulation of the wrist

- Lidoderm patches

* Recommended by the American Academy of Neurology (diuretics, only possibly)

\section{UNANSWERED RESEARCH QUESTIONS}

- No universally agreed case definition exists

- What is the long term outcome of local steroid injection?

- How many times and how frequently may steroid injection be repeated?

- What is the mode of action of steroids in CTS?

- Does hand use in certain occupations cause CTS?

-What factors predict response to treatment?

satisfactory symptom relief from this measure alone,$^{21}$ and splints have the advantage of being inexpensive (£3.50 each) and without serious adverse effects.

\section{Steroids}

Carpal tunnel syndrome has been shown to respond to both systemic steroids and to local steroids given at (or near) the wrist by either injection or iontophoresis (transdermal delivery driven by an electric field). The side effects of oral steroids preclude their routine use for CTS, but local steroid injection has no discernible systemic effects and a very low incidence of local complications. Although median nerve damage from intraneural injection has been reported in eight cases, pooling the reported trials of steroid injection yields a total of over 3000 injections performed without serious complications, and the risk may be estimated at $<0.1 \%$ in competent hands.

The initial response rate to a single steroid injection is about 70\%, but relapse is common. No adequate long term studies exist to allow precise quantification of the relapse rate beyond the first few months. The most pessimistic estimates suggest that $92 \%$ may have relapsed by two years. ${ }^{22}$ At the other extreme is a series in which half of injected patients remain in remission at seven years. No evidence is available to guide policy on treatment after relapse following a successful first injection, though it is common practice to inject a second or sometimes third time, and there are anecdotal reports of patients maintained long term on repeated injections.

\section{Surgery}

Carpal tunnel decompression, usually performed as a day case under local anaesthesia, is considered the definitive treatment. However, although it provides permanent and complete cure in most cases, it is not without risk. A survey of over 4000 patients having surgery under usual NHS circumstances found that about two years after surgery, only 75\% considered the operation an unqualified success and $8 \%$ thought that they were worse off. ${ }^{23}$ Although papers in the literature devoted to "recurrent CTS" are numerous, true recurrence, after successful initial surgery, is rare. It may be more common after endoscopic surgery. ${ }^{24}$ Most reports in fact relate to primary failure of the operation to relieve symptoms. Such failures are mostly attributable to misdiagnosis (the symptoms actually being due to other causes, whether or not 


\section{SUMMARY POINTS}

Carpal tunnel syndrome is the commonest cause of nocturnal hand paraesthesias and

should be suspected in any patient with this symptom, whatever age

Initial treatment should be with neutral angle splinting at night; milder cases may be treated with local steroid injection before further investigation

Refer more severe cases-those with thenar weakness, sensory deficit, and daytime symptoms-for nerve conduction studies and possible surgery

Either open or endoscopic surgery may be used depending on availability of local expertise

there is a median lesion at the wrist), surgical errors (the commonest being failure to fully divide the transverse carpal ligament ${ }^{25}$ ), and delay of treatment to a point when median nerve function is beyond recovery. A small minority are the result of more unpredictable surgical complications, inadvertent nerve and vessel lacerations, infections, painful scarring, and complex regional pain syndrome.

Although endoscopic methods of carpal tunnel decompression have been popular in recent years, long term outcomes do not differ significantly between the traditional and endoscopic approaches, and even the possible advantage of an earlier return to use of the hand after endoscopic surgery seems limited to a few days only. ${ }^{4}$ Despite the greater technical difficulty of endoscopic surgery, it does not seem to be associated with greater incidence of serious complications. However, most published series of endoscopic procedures have been performed by enthusiasts for the technique who have achieved considerable technical proficiency. Some caution should therefore be shown before the widespread adoption of these methods by the occasional carpal tunnel surgeon.

Patients with CTS having surgery before reaching grade 6 on the Canterbury scale whose symptoms do not respond to surgery should have repeat nerve conduction studies performed within three months, and if these show no change or deterioration, re-exploration should be performed, particularly looking for incomplete ligament section. Imaging studies may also be able to show this surgical mistake, but this has not yet been systematically reported.

Overall, patients whose CTS symptoms are significantly troublesome and who have mild or moderate impairment of median nerve function should be offered splinting and local steroid injection. Patients failing such conservative management and those who present at a later stage with objective

\section{ADDITIONAL EDUCATIONAL RESOURCES}

For health professionals

- Richard B Rosenbaum, Jose L Ochoa. Carpal tunnel syndrome and other disorders of the median nerve. 2nd ed. Boston, MA: Butterworth Heinemann, 2002.

For patients

Carpal Tunnel Syndrome Fact Sheet from the US National Institute of Neurological Disorders and Stroke (www.ninds.nih.gov/disorders/carpal_tunnel/ detail_carpal_tunnel.htm) neurological signs or delayed motor conduction on nerve conduction systems should be offered the option of surgical decompression. All should be advised of the potential risks of the different treatments.

Contributors: JDPB is the sole contributor

Competing interests: None declared.

Provenance and peer review: Commissioned and externally peer reviewed.

1 Practice parameter for carpal tunnel syndrome (summary statement). Report of the Quality Standards Subcommittee of the American Academy of Neurology. Neurology 1993;43:2406-9.

2 Gelberman RH, Hergenroeder PT, Hargens AR, Lundborg GN, Akeson WH. The carpal tunnel syndrome: a study of carpal canal pressures. J Bone Joint Surg 1981;63A:380-3.

3 Ettema AM, Amadio PC, Zhao C, Wold LE, An K-N. A histological and biochemical study of the subsynovial connective tissue in idiopathic carpal tunnel syndrome. J Bone Joint Surg 2004;86A:1458-66.

4 Hakim AJ, Cherkas L, El Zayat S, MacGregor AJ, Spector TD. The genetic contribution to carpal tunnel syndrome in women: a twin study. Arthritis Rheum 2002;47:275-9.

5 Bland JDP. The relationship of obesity, age, and carpal tunnel syndrome: more complex than was thought? Muscle and Nerve 2005;32:527-32.

6 Atroshi I, Gummesson C, Johnsson R, Ornstein E, Ranstam J, Rosen I. Prevalence of carpal tunnel syndrome in a general population. JAMA 1999;282:153-8.

7 Bland JDP, Rudolfer SM. Clinical surveillance of carpal tunnel syndrome in two areas of the United Kingdom, 1991-2001. J Neurol Neurosurg Psychiatry 2003;74:1674-9.

8 Blumenthal S, Herskovitz S, Verghese I. Carpal tunnel syndrome in older adults. Muscle and Nerve 2006;34:78-83.

9 Bland JDP. The value of the history in the diagnosis of carpal tunnel syndrome. J Hand Surg 2000;25B:445-50.

10 Mondelli M, Passero S, Giannini F. Provocative tests in different stages of carpal tunnel syndrome. Clin Neurol Neurosurg 2001;103:178-83.

11 Bindra RR, Evanoff BA, Chough LY, Cole RJ, Chow JCY, Gelberman RH. The use of routine wrist radiography in the evaluation of patients with carpal tunnel syndrome. J Hand Surg 1977;22A:115-9.

12 De Rijk MC, Vermeij FH, Suntjens M, van Doom PA. Does a carpal tunnel syndrome predict an underlying disease? J Neurol Neurosurg Psychiatry 2007;78:635-7.

13 Jablecki CK, Andary MT, Floeter MK, Miller RG, Quartly CA, Vennix MJ, et al. Practice parameter: electrodiagnostic studies in carpal tunnel syndrome. Neurology 2002;58:1589-92.

14 Bland JDP. Do nerve conduction studies predict the outcome of carpal tunnel decompression? Muscle Nerve 2001;24:935-40.

15 Wong SM, Griffith JF, Hui ACF, Lo SK, Fu M, Wong KS. Carpal tunnel syndrome: diagnostic usefulness of sonography. Radiology 2004:232(1):93-99.

16 Verdugo RJ, Salinas RS, Castillo J, Cea JG. Surgical versus non-surgical treatment for carpal tunnel syndrome. Cochrane Database Syst Rev 2003;(3):CD001552.

17 Marshall S, Tardif G, Ashworth N. Local corticosteroid injection for carpal tunnel syndrome. Cochrane Database Syst Rev 2007;(2) CD001554.

18 Scholten R, Bouter LM, Gerritsen A, Uitdehaag BM, de Vet HCW, van Geldere D Surgical treatment options for carpal tunnel syndrome. Cochrane Database Syst Rev 2004; (1):CD003905.

19 O'Connor D, Marshall S, Massy-Westropp N. Non-surgical treatment (other than steroid injection) for carpal tunnel syndrome. Cochrane Database Syst Rev 2003;(1):CD003219.

20 Padua L, Padua R, Aprile I, Pasqualetti P, Tonali P. Multiperspective follow-up of untreated carpal tunnel syndrome: A multicenter study. Neurology 2001;56:1459-66.

21 Gerritsen AAM, de Vet HCW, Scholten RJPM, Bertelsmann FW, de Krom MCTFM, Bouter LM. Splinting vs surgery in the treatment of carpal tunnel syndrome: a randomised controlled trial. JAMA 2002;288:1245-51.

22 Girlanda P, Dattola R, Venuto C, Mangiapane R, Nicolosi C, Messina C. Local steroid treatment in idiopathic carpal tunnel syndrome: short and long term efficacy. J Neurol 1993;240:187-90.

23 Bland JDP. Carpal tunnel syndrome. Curr Opin Neurol 2005;18:581-5.

24 Concannon MJ, Brownfield ML, Puckett CL. The incidence of recurrence after endoscopic carpal tunnel release. Plast Reconstr Surg 2000;105:1662-5.

25 Assmus H. Korrektur und Rezidiveingriffe beim Karpaltunnelsyndrom. Bericht uber 185 Nachoperationen [Correction and reintervention in carpal tunnel syndrome. Report of 185 reoperations]. Nervenarzt 1996;67:998-1002. 